\title{
AN INTRODUCTION TO SIMULATION AND OPTIMIZATION
}

\author{
J. W. BANDLER \\ Group on Simulation, Optimization and Control \\ and Department of Electrical Engineering
}

McMaster University, Hamilton, Canada

Irvited Poper

\section{Abstract}

A review of recent work in simulation and optimization is made with the aim of introducing the designer to the benefits of automating optimal design procedures and to indicate limitations imposed by the current state of the art.

\section{Introduction}

This paper is directed to the engineer interested in using computer aids to modeling and design, and considering the application of optimization techniques. Limitations on the size and scope of problems which can be approached from the optimization point of view as imposed by the current state of the art are also indicated.

It seems that the gap between theoretical developments and their practical implementation is in danger of widening. With the plethora of literature in optimization methods and computer-aided circuit design, particularly articles laying claim to superiority of technique, a confused impression is created.

With these thoughts in mind, the author will attempt to direct the microwave reader to work which appears relevant, useful, instructive or stimulating within the domain of activity of the respective authors.

\section{Review}

It is felt that Calahan's book on computer-aided network design 1 is a good indicator of current trends and possible future developments in computer based circuit and system design techniques and philosophies. The collection of articles ${ }^{2}$ considered by Director to be benchmarks in simulation and optimization is also recommended, again not so much for the details as for its point of view. Szentimai's reprint volume ${ }^{3}$ deals with various aspects of filter design, and appears representative of numerical advances in that area.

Complementary survey articles on optimization techniques are those by Bandler 4 and Charalambous 5 . Also appearing in the IEEE Transactions on Microwave Theory and Techniques and somewhat complementary in the areas of simulation and sensitivity analysis are papers by Bandler and Seviora 6 and Monaco and Tiberio ${ }^{7}$. A pragmatic article of particular interest to microwave designers is one by Per1man and Gelnovatch 8 .

\section{Analysis}

Effort is being directed at solving larger systems more efficiently. See, for example, Wexler et $\mathrm{a}^{9}{ }^{9}$ and others 10,11. As far as engineering design is concerned, it is important to stress that it is generally inefficient to put a conventional simulation program into an optimization loop without taking certain things into account. Assuming, for example, that the program exploits sparsity in the computations the question of efficient computation of the effects of parameter changes (indispensable to design) arises. In general, for economical and physical reasons, not all possible design variables or degrees of freedom are always utilized. Setting up the necessary equations and recomputing the entire response every time a relatively small number of parameters is changed will result in a much larger computing bill than is necessary. In considering the value of an analysis routine for design purposes, then, the manner in which the effects of component variation are handled is crucial.

\section{Sensitivity}

A much debated topic in the circuit literature, particularly in time-domain analysis, efficient sensitivity evaluation is a cornerstone to automatic design ${ }^{2}$. Branin12 has dispelled some of the mystique shrouding the adjoint network method 2,6 by a compact, abstract presentation. The adjoint network approach whereby, for example, the first-order sensitivities of the output of a circuit may be efficiently evaluated with respect to all designable components using the results of only two circuit analyses has, however, been a powerful motivating force.

Extensions and applications of the adjoint network concept abound in the literature 7,13 . In the frequency domain for linear circuits, at least, it appears that, by suitable mathematical manipulations, higher-order sensitivities ${ }^{14}, 1$ arge-change sensitivities 15 , sensitivities with respect to frequencylo etc., are available relatively efficiently by suitable programming. The most widely acclaimed optimization methods ${ }^{5}$, however, require only first derivatives. Furthermore, the value of second- and higher-order sensitivities at points possibly far from the optimum has not been estab1ished.

\section{Formulation}

There appear to be two principal approaches to the formulation of design objectives. On the one hand, some designers attempt to approximate ideal performance specifications which, by definition, are unattainable. This approach requires the least preparation of the problem, but the results tend to be somewhat ambiguous in the context of meeting specifications and subsequent assignment of tolerances. On the other hand, more insight can be brought to bear if design problems are cast in the form of meeting or exceeding realistic performance specifications 4 . One can go a step further, exploiting more fully one's prior knowledge or insight into the problem at hand, by devising artificial specifications ${ }^{17}$ in an attempt to anticipate more closely the actual optimum performance realizable by the configuration and thereby permit its more rapid evaluation. Optimal assignment of manufacturing tolerances appears to be more well-defined in the context of realistic specifications.

\section{Objectives}

The ubiquitous least squares objective 2,18 ,usually employed in conjunction with error-prone data or ideal 
specifications in the context, for example, of modeling or design, respectively, is probably the simplest to implement. Particularly in filter design, however, nonEuclidean measures of error have been widely applied historically. See, for example, Szentirmai ${ }^{3}$. Numerical approximation methods for minimax (Chebyshev) or near minimax solutions, contrary to prevailing assumptions, can, for all practical purposes be realized almost as easily as least squares solutions 19 . On paper, at least, they produce more impressive-looking responses.One reason is that one or more trial runs are usually required in practice to verify a solution. Once a run,for example, using a least squares objective has been performed, sufficient information about the properties of the problem is often available to allow one to subsequently force at least a near minimax solution with relatively little additional effort ${ }^{19}$.

\section{Algorithms}

It is known that a well-conditioned problem in terms of selection of a well-behaved objective function and nonredundant variables which have been properly scaled allows the conventional steepest descent method to perform adequately. The Newton method, which may be viewed as steepest descent with respect to a different norm 20 is generally less sensitive to scaling but, unlike steepest descent, is affected by the properties of the second derivatives and convexity.

Modem gradient methods 21,22 attempt to overcome the limitations of the basic steepest descent and Newton methods, as do analogous methods in the minimax optimization of a set of functions $19,23,24$. In minimax problems, in particular, classical assumptions about the number or character of the equal (or active) extrema vis a vis the number of independent variables need not and, in general, do not hold.

Current efforts in optimization 20 are directed at developing robust algorithms, however, anticipation and alleviation of ill-conditioning, where possible, is desirable.

\section{Centering}

Centering a design usually implies the process of finding a nominal design somehow influenced by manufacturing tolerances and, possibly, post production tuning 25,26 . The procedure may involve optimal assignment of component tolerances, maximization of production yield, design subject to a specified yie1d, etc. The problem could be a worst-case one with design variables assumed independent; it might involve correlated elements, statistical distributions, and so on. A numbe of relevant works will provide the interested reader with further details 27 . It should be emphasized that, in general, all design parameters: nominal values, tolerances, tuning ranges and so on will interact in defining an optimal design 25,26. A solution obtained from a least squares or minimax approximation in the usual sense does not necessarily provide the best nominal values. The centering problem is generally significant1y more expensive to solve, requiring careful preparation.

\section{Software}

An excellent survey of both available and proprietary genera1 purpose software for circuit designers has been made by Kap $1 a^{18}$. The article, however, appears limited to developments in the U.S. and probably places undue emphasis on least squares objectives. A number of optimization programs with documentation is available from the present author 28 . Two collections 29,30 of reprints, reports, notes and programs should also be mentioned. Documented listings of very useful optimization programs are also available from the U.K. Atomic Energy Research Establishment 31 , and the Numerical Optimization Centre 32 . See also pp.242-243 of Gi11 and Murray 20 .

Should one use a commercially available analysis and design package, for example, through a time-sharing facility? It is felt that current optimization features in these packages are generally weak, so that their use will probably be expensive in the long run.

New algorithms or packages should be tested on suitable examples and compared with respect to features, flexibility, ease of use, convergence to known solutions, memory required and running times. This is particularly appropriate in optimal design where, over an extended period of use, enormous numbers of simulations might be required.

Techniques which appear different may sometimes be alternative implementations of the same basic algorithm ${ }^{5}$. This is, understandably, often not realized at the time by the proponents of the techniques. As the state of the art advances, unification takes place and the techniques can be put into better perspective. See a1 so Branin 12 and Bonfatti et a 11 .

\section{Conclusions}

Having assimilated the essential past achievements (regrettably inadequately referenced because of limited space) where might one find indicators of possible new developments? Three additional recent works may be singled out: an advance in minimax algorithms where derivatives are not required 33 , an advance in efficient design in the time domain employing sensitivity information 34 , and an advance in centering which takes account of many uncertainties relevant to the microwave area 35. A number of sessions at this year's IEEE International Symposium on Circuits and Systems (Munich, Germany, Apr. 1976) promise further achievements in simulation and optimization in all areas covered by this paper ${ }^{36}$.

\section{References}

${ }^{1}$ D.A. Calahan, Computer-Aided Network Design (Revised Edition). New York: McGraw-Hi11, 1972.

${ }^{2}$ S.W. Director, Ed., Computer-Aided Circuit Design: Simulation and Optimization. Stroudsburg, Penn.: Dowden, Hutchinson and Ross, 1973.

${ }^{3}$ G. Szentirmai, Ed., Computer-Aided Filtex Design. New York: IEEE Press, 1973.

${ }^{4} \mathrm{~J} . \mathrm{W}$. Bandler, "Optimization methods for computer-aided design," IEEE Trans. Microwave Theory Tech., vol.MTT-17, Aug. 1969, pp.533-552.

${ }^{5}$. Charalambous, "A unified review of optimization," IEEE Trans. Microwave Theory Tech., vo1. MTT-22, Mar. 1974, pp. 289-300.

${ }^{6}$ J.W. Bandler and R.E. Seviora, "Current trends in network optimization," IEEE Trans Microwave Theory Tech., vo1. MTT-18, Dec. 1970, pp. 1159-1170.

7V.A. Monaco and P. Tiberio,"Computer-aided analysis of microwave circuits," IEEE Trans. Microwave Theory Tech., vo1.MTT-22, Mar. 1974, pp. 249-263.

${ }^{8}$ B.S. Perlman and V.G. Gelnovatch, "Computer aided design, simulation and optimization," in Advances in 
Microwaves vo1.8, L. Young and H. Sobo1, Eds. New York: Academic Press, 1974.

${ }^{9}$ A. Wexler et a1, "Solution of large, sparse systems in design and analysis", IEEE Int. Microwave Symp. Digest (Palo Alto, Calif., May 1975).

${ }^{10}$ G.D. Hachte1, R.K. Brayton and F.G. Gustavson, "The sparse tableau approach to network analysis and design", IEEE Trans. Circuit Theory, vol. CT-18, Jan. 1971, pp.101-113.

${ }^{11}$ F. Bonfatti, V.A. Monaco and P. Tiberio, "Microwave circuit analysis by sparse-matrix techniques", IEEE Trans. Microwave Theory Tech., vol.MTT-22, Mar. 1974, pp.264-269.

12F.H. Branin, Jr., "Network sensitivity and noise analysis simplified", IEEE Trans. Circuit Theory, vo1. CT-20, May 1973, pp.285-288.

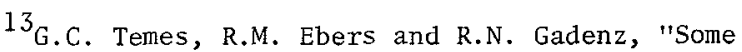
applications of the adjoint network concept in frequency domain analysis and optimization", Computer Aided Design, vol. 4, Apr. 1972, pp. 129- $\overline{134}$.

${ }^{14}$ A.K. Seth and P.H. Roe, "Hybrid formulation of explicit formulae for higher order network sensitivities", IEEE Trans. Circuits Syst., vol. CAS-22, May 1975, pp.475-478.

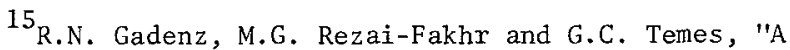
method for the computation of large tolerance effects", IEEE Trans. Circuit Theory, vol. CT-20, Nov.1973, pp.704-708.

${ }^{16}$ J.W. Bandler, M.R. M. Rizk and H. Tromp, "Efficient calculation of exact group delay sensitivities", IEEE Trans. Microwave Theory Tech., vo1. MTT-24, Apri1 1976.

${ }^{17} \mathrm{C}$. Charalambous and J.W. Bandler, "New algorithms for network optimization", IEEE Trans. Microwave Theory Tech., vo1. MTT-21, Dec. 1973, pp.815-818.

${ }^{18}$ G. Kaplan, "Computer-aided design", IEEE Spectrum, vol.12, Oct.1975, pp.40-47.

${ }^{19}$ J.W. Bandler, C. Charal ambous, J.H.K. Chen and W.Y. Chu, "New results in the least pth approach to minimax design", IEEE Trans. Microwave Theory Tech., vo1. MTT-24, Feb. 1976 .

20p.E. Gill and W. Murray, Eds., Numerical Methods for Constrained Optimization. New York: Academic Press, 1974 .

$21_{R}$. F1etcher, "Fortran subroutines for minimization by quasi-Newton methods", Atomic Energy Research Establishment, Harwe11, England, Report AERE-R7125,1972.

22p.E. Gi11 and W. Murray, "Quasi-Newton methods for unconstrained optimization", J.Inst.Math.Applics., vo1.9, 1972, pp.91-108.
${ }^{23}$ K. Madsen, H. Schjaer-Jacobsen and J. Voldby, "Automated minimax design of networks", IEEE Trans. Circuits Syst., vol. CAS-22, Oct.1975, pp.791-796.

${ }^{24}$ C. Charalambous and A.R. Conn, "Optimization of microwave networks", IEEE Trans. Microwave Theory Tech., vo1. MTT-23, Oct. 1975, pp.834-838.

${ }^{25}$ J.F. Pine1, K.A. Roberts and K. Singha1, "Tolerance assignment in network design", proc. IEEE Int. Symp. Circuits Syst. (Newton, Mass., Apr. 1975),pp.317-320.

${ }^{26}$ J.W. Bandler, P.C. Liu and H. Tromp, "A nonlinear programming approach to optimal design centering, tolerancing and tuning", IEEE Trans. Cixcuits Syst., vo1. CAS-23, Mar. 1976.

27 "Tolerance assignment in network design", Special Session, Proc. IEEE Int. Symp. Circuits Syst. (Newton, Mass., Apr. 1975), pp.317-336.

${ }^{28}$ Group on Simulation, Optimization and Control, Faculty of Engineering, McMaster University, Hamilton, Canada L8S $4 \mathrm{~L} 7$.

${ }^{29}$ W. Kinsner, Ed., "Notes on numerical methods in engineering analysis and design of fields, circuits and systems", Faculty of Engineering, McMaster Univ., Hamilton, Canada, Report SOC-93, June 1975.

${ }^{30} \mathrm{~J} . W$. Bandler, Ed., "Notes on numerical methods of optimization with app1ications in optimal design", Faculty of Engineering, McMaster Univ., Hamilton, Canada, Report SOC-113, Nov.1975.

${ }^{31}$ Computer Sciences and Systems Division, Atomic Energy Research Establishment, Harwe11, Eng1 and 0x11 ORA.

${ }^{32}$ Numerical Optimization Centre, Hatfield Polytechnic, Hatfield, Hertfordshire, England.

${ }^{33}$ K. Madsen, O. Nielsen, H. Schjaer-Jacobsen and L. Thrane, "Efficient minimax design of networks without using derivatives", IEEE Trans. Microwave Theory Tech., vol. MTT-23, oct. 1975, pp.803-809.

${ }^{34}$ R.K. Brayton and S.W. Director, "Computation of delay time sensitivities for use in time domain optimization", IEEE Trans. Circuits Syst., vol. CAS22, Dec. 1975, pp. 910-920.

$35 \mathrm{~J}$.W. Bandler, P.C. Liu and H. Tromp, "Integrated approach to microwave design", IEEE Trans. Microwave Theory Tech., to appear.

${ }^{36}$ Regular and Special Sessions, IEEE International Symposium on Circuits and Systems, Munich, Germany, Apr. 1976. 